\title{
Pyruvate in the Correction of Intracellular Acidosis: A Metabolic Basis as a Novel Superior Buffer
}

\author{
Fang Qiang Zhou \\ Fresenius Dialysis Centers, Chicago, III., USA
}

\author{
Key Words \\ Pyruvate - Lactate - Bicarbonate - Glucose • Ischemia • \\ Intracellular $\mathrm{pH} \cdot$ Acidosis
}

\begin{abstract}
The review focuses on biochemical metabolisms of conventional buffers and emphasizes advantages of sodium pyruvate (Pyr) in the correction of intracellular acidosis. Exogenous lactate (Lac) as an alternative of natural buffer, bicarbonate, consumes intracellular protons on an equimolar basis, regenerating bicarbonate anions in plasma while the completion of gluconeogenesis and/or oxidation occurs via tricarboxylic-acid cycle in mitochondria mainly in liver and kidney, or heart. The general assumption that Lac is 'metabolized to bicarbonate' in liver to serve as a buffer has been questioned. Pyr as a novel buffer would be superior to conventional ones in the correction of metabolic acidosis. Several likely biochemical mechanisms of Pyr action are discussed. Experimental evidence, in vivo, strongly suggested that Pyr would be particularly efficient in the correction of severe acidemia: type A lactic acidosis, hypercapnia with cardiac arrest, and diabetic and alcoholic ketoacidosis in animal experiments and clinic settings. Because of its multi-cytoprotec-
\end{abstract}

The opinions or assertions contained herein are the author's private view and are not to be construed as reflecting the view of Fresenius Dialysis Centers at Chicago, USA.
(C) 2005 S. Karger AG, Basel 0250-8095/05/0251-0055\$22.00/0

Fax +4161306 1234 E-Mail karger@karger.ch www.karger.com www.karger.com/ajn tion, Pyrs not only correct acidosis, but also benefit the underlying dysfunction of vital organs. In addition, Pyr is also a potential buffer component of dialysis solutions. However, the instability of Pyr in aqueous solutions restricts its clinical applications as a therapeutic agent. Attempts to create a stable Pyr preparation are needed.

Copyright (c) 2005 S. Karger AG, Basel

Since 1970s, pyruvate (Pyr) has become increasingly attractive in the protection of dysfunctional vital organs, particularly in myocardial ischemia and reperfusion injury, pointing to a potential therapeutic value for the dysfunctional myocardium [1-3]. Also, findings strongly indicated that Pyr would be a novel buffer in many clinic settings [4-6]. This review discusses its distinctive effects on intracellular $\mathrm{pH}$ ( $\mathrm{pHi}$ ), proposing that $\mathrm{Pyr}$ may be superior to lactate ( $\mathrm{Lac}$ ) and bicarbonate in the correction of severe intracellular acidosis.

\section{Lactate Metabolism and Effects on pHi and Lactic Acidosis}

Lac has been traditionally accepted as the best replacement for bicarbonate in the treatment of metabolic acidosis since early 1930s [7]; it has since become part of solutions for dialysis and continuous renal replacement therapy for several decades. 
However, the widely propagated theory that Lac is 'metabolized to bicarbonate' on an equimolar basis in liver represents a simplification. In fact, Lac is an end product of anaerobic glycolysis with Pyr as its only outlet. As an alkalizer, it must be converted to Pyr by lactic dehydrogenase (LDH) in most mammalian tissues, which in turn can either be completely oxidized to $\mathrm{CO}_{2}$ and $\mathrm{H}_{2} \mathrm{O}$ in the mitochondria mainly in liver, kidney, heart and brain for the titration of a proton or enter gluconeogenesis from two Lac anions in the mitochondria and cytosol of liver and kidney for the titration of two protons. Both Lac oxidation and Lac-based gluconeogenesis are a mitochondrial tricarboxylic-acid cycle (TCA cycle)-dependent pathway. The overall reactions can be described as shown in equations 1 and 2; whichever route of Lac disposal is followed, one proton $\left(\mathrm{H}^{+}\right)$is consumed per Lac anion metabolized $\left(\mathrm{Lac}^{-}: \mathrm{H}^{+}=1: 1\right)$ :

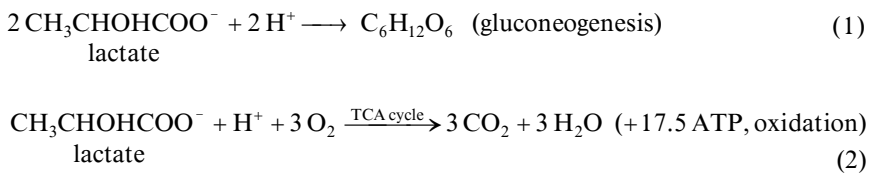

The consumption of a proton in cytosol effectively means the regeneration of a bicarbonate anion, thus raising $\mathrm{pHi}$ and preserving the blood bicarbonate level stoichiometrically $[8,9]$.

The uptake of Lac or Pyr by cells and the entry of Pyr into mitochondria depend on the $\mathrm{Lac}^{-} / \mathrm{H}^{+}$cotransport system, monocarboxylate transporters (MCT, fig. 1) with $\mathrm{H}^{+}\left(\left[\mathrm{H}^{+}\right]\right.$in fig. 1$)$ flux in symport. The system is located in the cytoplasma membrane and inner membrane of mitochondria. The oxidation of exogenous Lac to Pyr by $\mathrm{LDH}$ is $\mathrm{NAD}^{+}$-dependent in cytosol, which releases two hydrogen ions with one $\left(\mathrm{H}^{+}\right.$in fig.1) left in the hydrogen pool of cytosol as shown in equation 3. Both MCT-transported and Lac-released protons initially decrease $\mathrm{pHi}$ $[10,11]$, while the other hydrogen ion (negative hydrogen or hydride ion) released from $\mathrm{Lac}$ reduces cytosolic $\mathrm{NAD}^{+}$ $\left(\left[\mathrm{NAD}^{+}\right] \mathrm{c}\right)$ to NADH $([\mathrm{NADH}] \mathrm{c})$. Such a decrease of reducing equivalents has been called cellular 'pseudohypoxia'. In aerobic conditions, $\left[\mathrm{NADH}_{2}\right] \mathrm{c}([\mathrm{NADH}+$ $\left.\mathrm{H}^{+}\right] \mathrm{c}$ ) may be transferred into mitochondria and oxidized with the generation of $1 \mathrm{H}_{2} \mathrm{O}$ and 2.5 ATP. Thus, the proton released from Lac is eventually consumed. The administration of Lac raises the Lac/Pyr ratio and decreases the $\left[\mathrm{NAD}^{+} / \mathrm{NADH}\right] \mathrm{c}$ ratio in cytosol, which may critically inhibit glycolysis at the level of glyceraldehyde3-phosphate dehydrogenase (G-3-PD) [12]. It also reduces the glycolytically derived ATP production that is vital for sustaining cellular functions in anaerobic conditions. Pyr oxidative decarboxylation, then, is catalyzed by the Pyr dehydrogenase complex (PDC) in mitochondria, coupled with the reduction of mitochondrial $\mathrm{NAD}^{+}$ $\left(\left[\mathrm{NAD}^{+}\right] \mathrm{m}\right)$ to $[\mathrm{NADH}] \mathrm{m}$, yielding acetyl-CoA. It depends on oxygen as an electron acceptor in mitochondria that one pair of $\left[\mathrm{NADH}_{2}\right] \mathrm{m}\left(\left[\mathrm{NADH}+\mathrm{H}^{+}\right] \mathrm{m}\right)$ as well as transferred $\left[\mathrm{NADH}_{2}\right] \mathrm{c}$ is oxidized through the electron transfer chain, consuming the two hydrogen ions of $\left[\mathrm{NADH}_{2}\right] \mathrm{m}$ with $1 \mathrm{H}_{2} \mathrm{O}$ and 3 ATP generations.

Catalyzed by PDC, one hydrogen (hydride) ion of $\left[\mathrm{NADH}+\mathrm{H}^{+}\right] \mathrm{m}$ is transferred via $\left[\mathrm{FADH}_{2}\right]$ from the reactive thiol group (-SH) of coenzyme A, HS-CoA, which does not affect $\mathrm{pHi}$; the other hydrogen ion (proton) is picked up in the hydrogen pool in the mitochondria matrix [13]. The overall reaction is the oxidation of imported proton via MCT and raises $\mathrm{pHi}$ as reported in cardiomyocytes of ischemic reperfused rabbit hearts and failing human myocardium $[10,11]$. Thus, for every exogenous Lac anion metabolized in aerobic conditions either by oxidative phosphorylation or with glyconeogenesis, one endogenous proton is consumed, leading to the preservation of blood bicarbonate levels on a 1:1 molar basis.

In the mitochondria of many cell lines, mainly hepatocytes, carbonic anhydrase isoenzyme (CA V) exists, which catalyzes hydration $\left(-\mathrm{HCO}_{3}\right.$ or $\mathrm{H}_{2} \mathrm{CO}_{3}$ ) of $\mathrm{CO}_{2}$ and $\mathrm{H}_{2} \mathrm{O}$. The bicarbonate so generated in the mitochondria is vital for the activity of bicarbonate-requiring Pyr carboxylase (PC) that is essential for the TCA cycle and gluconeogenesis $[14,15]$. Also, acetazolamide, an inhibitor of most CA isoenzymes including $\mathrm{CA} \mathrm{V}$, may decrease Lac-induced blood alkalinization and induce lactic acidosis [15]. Although these facts support that CA V plays an important role in the pHi modulation, they do not prove the general assumption that "the irreversible oxidation of Lac generates alkali from the conversion of $\mathrm{CO}_{2}$ to bicarbonate by CA', which then corrects metabolic acidosis $[16,17]$. The traditional concept that Lac as a buffer is 'metabolized to bicarbonate' in liver mitochondria catalyzed by CA V to correct acidosis lacks direct evidence. It is well known that the Lac oxidation induces cytotoxic effects in tissue ischemia or hypoxia, such as the depletion of reducing equivalents, inhibition of glycolysis and release of protons in cytosol. Hence, in hypoxic conditions, the administration of sodium Lac may aggravate, rather than attenuate, intracellular acidification if the ability of Pyr oxidation is limited and gluconeogenesis is suppressed in cells such as hepatocytes, cardiomyocytes and macrophages, which have abundant mitochondria. 


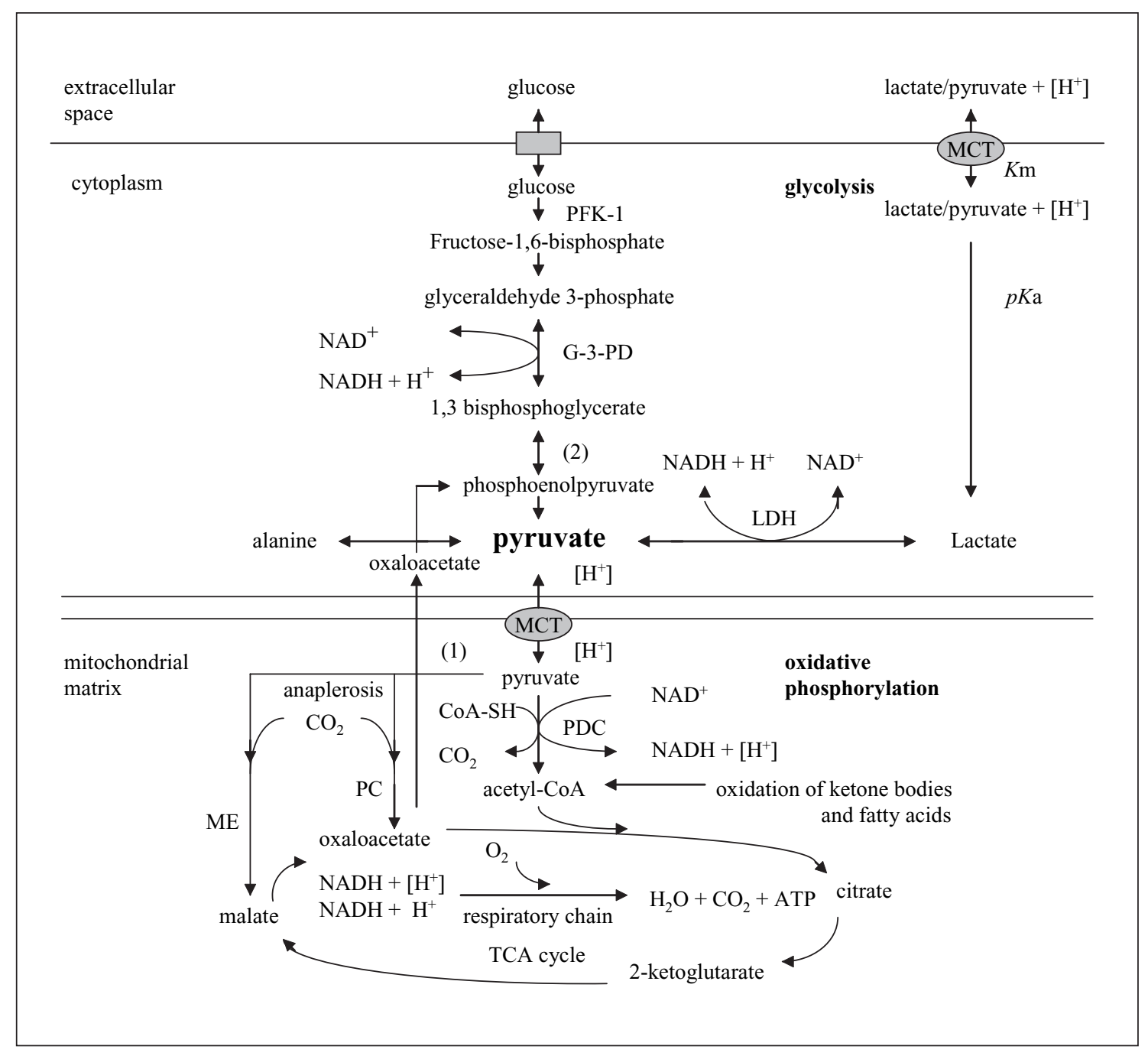

Fig. 1. Pyruvate metabolic pathways and their relationship with the consumption of protons in intracellular compartments. Pyr and/or Lac enter cytosol via the MCT system with an extracellular $\left[\mathrm{H}^{+}\right]$ flex in symport. The oxidation of Lac to Pyr with LDH releases an $\mathrm{H}^{+}$coupled with $\mathrm{NAD}^{+}$reduction to NADH in cytosol, inhibiting glycolysis at the G-3-DP step. Both MCT-imported $\left[\mathrm{H}^{+}\right]$and Lacreleased $\mathrm{H}^{+}$initially decrease $\mathrm{pHi}$, whereas the Pyr reduction to $\mathrm{Lac}$ consumes the $\left[\mathrm{H}^{+}\right]$, restoring $\mathrm{pHi}$ and glycolysis in anaerobic conditions. In aerobic conditions, the Pyr entry into mitochondria with $\left[\mathrm{H}^{+}\right]$yields $\mathrm{NADH}+\left[\mathrm{H}^{+}\right]$by PDC. Oxidative phosphorylation of both $\mathrm{NADH}+\left[\mathrm{H}^{+}\right]\left(\left[\mathrm{NADH}+\mathrm{H}^{+}\right] \mathrm{m}\right)$ and transferred NADH + $\mathrm{H}^{+}\left(\left[\mathrm{NADH}+\mathrm{H}^{+}\right] \mathrm{c}\right)$ generates ATP and $\mathrm{H}_{2} \mathrm{O}$, eventually consuming $\left[\mathrm{H}^{+}\right]$and $\mathrm{H}^{+}$, respectively. The overall oxidation of exogenous Pyr and/or Lac consumes endogenous $\left[\mathrm{H}^{+}\right]$on an equimolar basis, raising $\mathrm{pHi}$ and $\mathrm{pHe}$. Excess Pyr simultaneously activates the PDC activity and enhances anaplerotic pathways via PC, accelerating the TCA cycle flux and the $\left[\mathrm{H}^{+}\right]$consumption. Pyr-based gluconeo- genesis consumes an additional pair of $\left[\mathrm{NADH}+\mathrm{H}^{+}\right] \mathrm{c}$ at the step of G-3-DP in cytosol, compared with Lac-based gluconeogenesis. The preservation of a near physiologic $\mathrm{pHi}$ and the acceleration of TCA cycle by excess Pyr enhance the Lac oxidation in lactic acidosis and the oxidation of ketone bodies and fatty acids in ketoacidosis. The shuttle of $\left[\mathrm{NADH}+\mathrm{H}^{+}\right] \mathrm{c}$ transference from cytosol to mitochondria is not shown. See the text for details. $(1)=$ Gluconeogenesis-coupled reactions in mitochondria; $(2)$ = gluconeogenesiscoupled reactions in cytosol; G-3-PD = glyceraldehyde-3-phosphate dehydrogenase; $\mathrm{Km}=$ Michaelis constant $\mathrm{LDH}=$ lactic dehydrogenase; $\mathrm{MCT}=$ monocarboxylate transporters; $\mathrm{ME}=$ malic enzyme; $\mathrm{NAD}^{+}=$oxidized nicotinamide adenine dinucleotide; $\mathrm{NADH}=$ reduced nicotinamide adenine dinucleotide; $\mathrm{PC}=$ pyruvate carboxylase; $\mathrm{PDC}=$ pyruvate dehydrogenase complex; PFK-1 = phosphofructokinase- $1 ; p K a=$ dissociation constant; TCA cycle $=$ tricarboxylic-acid cycle. 
In cells with no or a few mitochondria (e.g., erythrocytes or neutrophils, etc.), exogenous Lac would just deteriorate the acidosis and depress cell function [18] even in normoxymia.

Hyperlactatemia and lactic acidosis could be corrected with dichloroacetate (DCA) or THAM [16, 19], but their values in clinic settings are not clear due to the negative survival rate and/or potential toxicity $[19,20]$. The efficacy of Lac therapy in patients with lactic acidosis has not been systemically evaluated. In principle, severe acidemia: Type A lactic acidosis, hypercapnia with cardiac arrest and diabetic ketoacidosis, which account for over $90 \%$ of severe acidosis in clinic settings with excess mortality [19], are contraindications of the Lac administration. The Lac infusion should be avoided in cases of elevated serum Lac. Although sodium Lac itself is almost obsolete in the treatment of clinical acidosis, several recent studies have demonstrated that lactated Ringer's solution $(28 \mathrm{mM})$ was beneficial in the resuscitation of massive hemorrhagic shock accompanied with lactic acidosis. It significantly improved acidosis and survival rates [17, 21-23]. In rat working hearts after hemorrhagic shock accompanied with lactic acidosis and impaired PDC activities, Lac at $8.0 \mathrm{mM}$ in the presence of palmitate in perfusates saturated with $95 \%$ oxygen improved cardiac efficiency [21]. Most likely the intracellular acidosis was attenuated although pHi data were not determined. The paradoxical effect of exogenous Lac on experimental lactic acidosis above has not been fully interpreted, but may be associated with several reasons: different species of models, early stage of shock, over $40 \mathrm{~mm} \mathrm{Hg}$ of mean arterial pressure of shock and anesthesia conditions [17, 21-23]. The above findings indicated that the TCA cycle function in vital organs remained, at least partially, intact for Lac oxidation [24]. Notably, Lac-based gluconeogenesis in liver and kidney may still be preserved in the early stage of shock $[25,26]$. In particular, kidney is as important a gluconeogenic organ as liver for Lac disposal. It is estimated that one-half of glucose production within the Lac Cori cycle takes place in the kidney cortex and Lac accounts for approximately 50\% of renal gluconeogenesis. The Lac uptake remains relatively stable in hemorrhagic shock dogs until blood loss reaches $40 \%$ of total blood volume $[26,27]$. While acidosis significantly depresses hepatic uptake of Lac, renal gluconeogenesis is markedly enhanced by ischemic injury or acidosis [26, 28] due to the rapid stimulation of phosphoenolpyruvate carboxykinase [29]. Further, a recent clinical investigation found that in patients with early postoperative cardiogenic shock accompanied with lactic acidosis, the clearance of exogenous Lac was not significantly altered, compared with controls [30]. In contrast, if shock despite causes in origin are severe [31] or combined with alcoholic intoxication [23], preexisting liver diseases, diabetes or multiple organ injuries, the Lac infusion most likely exacerbates lactic acidosis. Therefore, it should be taken into account in the deduction of Lac effects on acidosis in above-mentioned experimental results if the oxidative phosphorylation of TCA cycle or gluconeogenesis in vital organs was, at least in part, sustained during early shock (hypoxia) or recovered after shock (reoxygenation). On the other hand, because the anoxia (hypoxia) of tissues and the dysfunction of TCA cycle commonly coexist in lactic acidosis, exogenous Lac should be avoided in such clinical settings even in the early stage of shock if the patient is critically ill, preferably in consideration of a new buffer, Pyr, instead.

\section{Pyruvate Improvement of Intracellular Acidosis}

Pyr was initially used in mid-1970 to buffer intracellular hydrogen ions and improve redox status in cardiomyocytes. The administration of exogenous Pyr promisingly improved glycolysis that was depressed from ischemia-reperfusion injury, preserved cardiac contractile function and prolonged the survival time of ischemic swine hearts [32]. Also, $\operatorname{Pyr}(10 \mathrm{mM})$, as a sole exogenous substrate, significantly attenuated the $\mathrm{pHi}$ decrease in the perfused rat ischemic myocardium. It was first discovered in 1994 that animals treated with the Pyr infusion did not develop an anesthetic agent-induced mild metabolic acidosis presented in controls [33]. The finding confirmed that the intravenous Pyr would induce 'systemic alkalization' that was not emphasized in its conception until 1999 [34]. The comparison of Pyr with Lac on pHi in human cells was first conducted with neutrophils in 1995 [4]. In an acidic milieu, following a slight pHi decrease, Pyr $(40 \mathrm{~m} M)$ maintained neutrophilic pHi close to a physiological value, whereas an identical Lac level brought about a prompt and substantial pHi decline. The extracellular $\mathrm{pH}[\mathrm{pHe}]$ of mixture solutions with Pyr was significantly higher than that with Lac counterparts as well. A subsequent report confirmed the phenomenon [Perit Dial Int Abstracts, 1997;17(suppl 1):S33]. The impact of Pyr on systemic acidosis, however, at present has not been well appreciated [31], and potential mechanisms whereby Pyr preferably corrected severe acidosis have not been fully elucidated either $[6,34]$. There are several likely bio- 
chemical sites on which the metabolism of exogenous Pyr consumes intracellular protons, indicating that Pyr is of particular significance in the pHi modulation [32, 35-37] (fig. 1):

(1) By reducing to Lac with $\mathrm{LDH}$, one exogenous Pyr anion consumes one proton from the cytosolic hydrogen pool, raising $\left[\mathrm{NAD}^{+} / \mathrm{NADH}\right] \mathrm{c}$ and $\mathrm{pHi}$, as described in equation 3:

$$
\underset{\text { pyruvate }}{\mathrm{CH}_{3} \mathrm{COCOO}^{-}}+\mathrm{NADH}+\mathrm{H}^{+} \stackrel{\mathrm{LDH}}{\longrightarrow} \underset{\text { lactate }}{\mathrm{CH}_{3} \mathrm{CHOHCOO}^{-}}+\mathrm{NAD}^{+}
$$

The $\left[\mathrm{NADH} / \mathrm{NAD}^{+}\right] \mathrm{c}$ ratio by the law of mass action is the major determinant of reactive direction. For any given cellular level of $\mathrm{Pyr}$, the ratio determines what fraction of Pyr is converted to Lac. When mitochondrial oxidative reactions are impaired, the ratio rises, and the conversion of Pyr to Lac increases. Pyr inhibits glycolysis in aerobic conditions, however, it is of pivot importance that Pyr favors anaerobic glycolysis due to the increase of $\left[\mathrm{NAD}^{+} / \mathrm{NADH}\right] \mathrm{c}$ ratio and restoration of pH-sensitive phosphofructokinase (PFK-1) activity [5, $32,37]$. This would preserve glycolytic ATP production that is essential for maintaining sarcoplasmic membrane function, modulating intracellular protons [38, 39], and prolonging cell survival of vital organs during hypoxia [2, 40]. In the swine model of hemorrhagic shock manifested lactic acidosis, the intravenous infusion of Pyr further raised blood Lac levels, indicating that the Pyr reduction occurred in cytosol, but lowered the blood Lac/Pyr ratio and efficiently avoided or corrected lactic acidosis [6, 34, 41, 42].

Accordingly, Pyr 'systemic alkalization' proposed originally referred to this cytoplasmic reduction and the mitochondrial oxidation of exogenous Pyr [34]. In this respect, Pyr may also consume a proton in neutrophilic cytosol with the reduction to Lac in addition to its weaker buffering capacity, both raising neutrophilic pHi [4].

(2) By oxidizing into $\mathrm{CO}_{2}$ and $\mathrm{H}_{2} \mathrm{O}$ via the TCA cycle in mitochondria, Pyr anions also consume protons on an equimolar basis $\left(\mathrm{Pyr}^{-}: \mathrm{H}^{+}=1: 1\right)$ as equation 4 shows:

$\underset{\text { pyruvate }}{\mathrm{CH}_{3} \mathrm{COCOO}^{-}+\mathrm{H}^{+}+2 \frac{1}{2} \mathrm{O}_{2} \stackrel{\text { TCA cycle }}{\longrightarrow}} 3 \mathrm{CO}_{2}+2 \mathrm{H}_{2} \mathrm{O}$ (+15 ATP, oxidation) (4)

As mentioned above, the exogenous Pyr consumes endogenous protons stoichiometrically in both anaerobic glycolysis and aerobic oxidation, raising $\mathrm{pHi}$, and $\mathrm{Pyr}$ does not release a proton as Lac does, but merely consumes it in the oxidation. In addition, Pyr oxidizes with a less oxygen consumption rate, compared with Lac.
(3) Supraphysiologic doses of exogenous Pyr would greatly stimulate the activity of PDC although there has been no data from human beings yet. It has been demonstrated with various animal tissues in rat, rabbit, bovine and swine that Pyr at several millimolars simultaneously stimulated the PDC activity by inhibiting Pyr dehydrogenase kinase in association with the improvement of cardiac performance and cerebral and hepatic functions [6, 41-43].

(4) Although Lac may replenish the TCA cycle by the malate-aspartate shuttle, an anaplerotic pathway that transfers Lac-reduced $\left[\mathrm{NADH}_{2}\right] \mathrm{c}$ to mitochondria [21], it is with Pyr, other than Lac or other keto acids, that anaplerosis via carboxylation can be carried out by PC to oxaloacetate or by malic enzyme to malate. In a near physiological condition, Pyr cardoxylation may account for, in vivo, only $5 \%$ of the citrate formation in swine hearts [44]. However, it may increase as many as 7 times in perfused rat hearts [24]. The increased availability of Pyr as a substrate and the PDC stimulation together with enhanced anaplerotic pathways would greatly increase the TCA cycle flux and accelerate the consumption of protons and the ATP generation. In addition, anaplerosis pathways, per se, fix $\mathrm{CO}_{2}$ in mitochondria. Importantly, during acute acidosis Pyr may increase the $\mathrm{CO}_{2}$ fixation in the mitochondria of liver and kidney [45], which may be of significance in improving local hypercapnia in ischemic tissues.

(5) Gluconeogenesis is an energy-requiring process. It consumes equivalently 3 high-energy phosphate bonds ( 2 ATP and 1 GTP) for $1 \mathrm{~mol}$ of glucose synthesized from $1 \mathrm{~mol}$ of Lac. However, the experimental evidence in $\mathrm{pHi}$ within the rat hepatic lobule revealed that gluconeogenesis from Lac is a proton-consuming process [46]. Compared with one proton consumed by every Lac anion in Lac-based gluconeogenesis (equation 1), every Pyr anion uses two protons in Pyr-based gluconeogenesis $\left(\mathrm{Pyr}^{-}: \mathrm{H}^{+}\right.$ $=1: 2$ ). In the cytosolic process of gluconeogenesis, a pair of $\left[\mathrm{NADH}+\mathrm{H}^{+}\right] \mathrm{c}$ is required at the step of G-3-PD. It is supplied by the Lac oxidation to Pyr in Lac-based gluconeogenesis, but is additionally consumed in Pyr-based gluconeogenesis. Hence, Pyr-based gluconeogenesis in liver and kidney would be of particular significance in the preservation of a near physiologic arterial $\mathrm{pH}(\mathrm{pHa})$ in shock. In this regard, it was affirmed with rat hepatic intralobular mapping results that protons are consumed stoichiometrically when weak acids are converted into neutral products (glucose, $\mathrm{CO}_{2}$ and $\mathrm{H}_{2} \mathrm{O}$ ) [46]. The statement may be the case of Lac, other than the case of Pyr in terms of gluconeogenesis. 
As mentioned earlier, the entry of Lac or Pyr into cells is carried by a proton-linked MCT family, which includes seven isoforms in a range of mammalian cells. They exhibit distinct cellular localization and have slightly different properties. Isoforms act as $\mathrm{Lac}^{-} / \mathrm{H}^{+}$symport with a 1:1 stoichiometry. In contrast to the MCT in human neutrophils, which has a similar affinity for both Lac and Pyr [5], the isoforms of hepatocytes and cardiomyocytes in rats have much higher Michaelis constant $(\mathrm{Km})$ values for L-Lac than those for Pyr (4.7 and $2.74 \mathrm{~m} M$ vs. 1.3 and $0.2 \mathrm{~m} M$ ), respectively $[47,48]$. Thus, Lac may more readily induce a pHi decline than Pyr when high extracellular concentrations of both are comparable in human vital organs. On the other hand, Pyr has a weaker buffering capacity due to the dissociation constant ( $p \mathrm{Ka}$ ) of 2.49 , compared with Lac $p K a$ of 3.9 [4]. The weaker buffering capacity usually results in a higher $\mathrm{pH}$ value. Owing to these properties, equimolar doses of external Pyr and Lac are not expected to result in equivalent $\mathrm{pHi}$ reductions in human cells as shown in neutrophils [4]. In rat cardiomyocytes, the property of MCT Km was also considered in the Pyr effect on pHi [49].

Based on its biochemical and biophysical characteristics, Pyr may be valuable in the treatment of severe metabolic acidosis such as type A lactic acidosis, hypercapnia with cardiac arrest, diabetic ketoacidosis and alcoholic intoxication although no clinic data have been reported yet. In theory, Pyr with insulin might be a more efficient regime in the correction of diabetic ketoacidosis mainly due to their potential synergetic effects on the oxidation of ketone bodies. Pyr in the regime might be also valuable to restore the sensitivity of insulin receptors inhibited by severe acidosis and reduce the insulin dosage to avoid hypoglycemia. Moreover, because of its better consumption of intracellular protons in the absence of the direct production of $\mathrm{CO}_{2}$ in hypoxia, Pyr might be also beneficial in respiratory acidosis. However, these deductions require experimental and clinical validations.

\section{Pyruvate Superiority in the Correction of Acidosis}

\section{Pyr and Lac in the Correction of Metabolic Acidosis}

The effect of intravenous Pyr on acid-base balance was compared with equimolar Lac in anesthetized dogs in late 1970s. Lac infusion was associated with a significantly slower rate of bicarbonate level rising in comparison with Pyr, indicating that the additional metabolic steps are required [50]. The oxidation of Lac-reduced $\left[\mathrm{NADH}_{2}\right] \mathrm{c}$ may take place either in cytoplasm-coupled reactions associated with gluconeogenesis, or a mitochondrial route in the presence of oxygen. In addition to the difference of the proton consumption in gluconeogenesis processes between Pyr and Lac, the mitochondrial route is an indirect pathway, which depends on the reducing equivalent of carrier systems. The malate-aspartate shuttle is the major step involved in transporting $\left[\mathrm{NADH}+\mathrm{H}^{+}\right] \mathrm{c}$ into the mitochondria in cells of heart, liver and kidney. The shuttle operation is driven by the electrical potential across mitochondrial membrane. Thus, the oxidation of Lac to Pyr is an energy-dependent step and operates slowly (in minutes) in physiological conditions [51]. In perfused rabbit cardiomyocytes, the pHi recovery was delayed by $12 \mathrm{~min}$. with Lac-added perfusates compared with Pyr-added counterparts in the presence of DCA [10]. This rate-limited step, which would display while the $\left[\mathrm{NADH}_{2}\right] \mathrm{c}$ load is increased, may be another interpretation of the retardation of pHi recovery in addition to the elevated proton load, per se, from the Lac oxidation and the [NADH]c increment [10]. Although the ratio of $\left[\mathrm{NAD}^{+} / \mathrm{NADH}\right] \mathrm{c}$ determines the balance between Pyr and Lac (equation 3 ), the reaction is in favor of the direction from Pyr to $\mathrm{Lac}$ as the lesser rise in blood Pyr concentration with Lac infusion than in blood Lac level with equal Pyr infusion [50]. Several recent findings evidently supported that Pyr would be superior to Lac in the treatment of clinic metabolic acidemia including lactic acidosis. In the rat peritoneal dialysis model, preliminary results revealed that uremic acidosis was better corrected and the functions of peritoneal cells were more preserved with Pyr-buffered dialysates than with Lac counterparts [J Am Soc Nephrol Abstracts, 1998;9:236A; also Chin J Nephrol 2001;17: 365-368]. Also, in the rat model of severe hemorrhagic shock manifested lactic acidosis, commonly seen in a clinical setting, the intravenous infusion of Pyr improved, whereas Lac exacerbated, metabolic acidosis. Pyr Ringer's solution resulted in a higher blood base excess level and a lower blood Lac level, compared with Lac Ringer's counterparts [31]. The study also substantiated that Pyr, in vivo, significantly decreased the expression of apoptosis markers, whereas Lac increased the markers after resuscitation in rat lungs [31] as observed, in vitro, in human neutrophils and endothelial cells [5, 52]. Although mechanisms of Pyr attenuation of apoptosis in shock resuscitation are not fully understood, it is well recognized that acidosis may be a triggering factor $[5,53]$. Studies in hemorrhagic shock repeatedly confirmed that Pyr effectively prevented or corrected severe lactic acidosis in swine $[6,34,41,42]$. A human trial demonstrated that 
Pyr-fortified cardioplegia solutions provided cardioprotection superior to Lac-based counterparts during surgical cardiac arrest although pHi data were absent [54]. These findings strongly suggested that Pyr was preferable in the correction of intracellular acidosis in both anaerobic and aerobic conditions.

In brief, because of the prevalence of LDH in mammalian cells, Pyr as an oxidant improves $\mathrm{pHi}$ and cellular functions, which is opposite to the deleterious effects on $\mathrm{pHi}$ and cytotoxic characteristics of Lac as a reductant in molecular and cellular levels particularly in anaerobic conditions. Meanwhile, due to the Lac oxidation to Pyr with LDH, both of them also exhibit somewhat similar metabolic and biological effects including the modulation of $\mathrm{pHi}$ in aerobic conditions.

\section{Pyr and Bicarbonate in the Treatment of Metabolic} Acidosis in Cardiogenic Shock and Dialysis

Bicarbonate as a natural buffer was not questioned in the treatment of metabolic acidosis until two decades ago. The bicarbonate corrects acidosis through chemical neutralization, which depends on a fair respiratory function, whereas Pyr exerts a metabolic titration of intracellular acidosis without directly producing $\mathrm{CO}_{2}$. Bicarbonate often benefits patients with metabolic acidosis in the absence of tissue hypoxia (e.g., uremic, diarrheic, and renal tubular acidosis). However, patients with cardiac arrest or cardiogenic shock (or septic shock, hepatic failure and diabetic ketoacidosis, etc.) usually have impaired tissue oxygen delivery. In these circumstances, the primary causes of acidosis result from the accumulation of lactic acid, the proton release from ATP hydrolysis and the retardation of local tissue $\mathrm{CO}_{2}$ ('metabolic' hypercapnia). And limited glycolytic ATP production may lead to a progressive $\mathrm{pHi}$ decline to $<6.0$ in cardiomyocytes [19, 53]. Bicarbonate administration in hypoxic states does not correct the underlying tissue hypoxia and is generally not successful in improving the acidosis or patients' general conditions. It usually results in severe adverse effects, leading to the exacerbation of hypercapnia and lactic acidosis and further decrease of tissue $\mathrm{pHi}$ in liver, brain, skeletal muscle and red cells $[19,55]$. In the presence of high $P_{\mathrm{CO}_{2}}$ and high non-bicarbonate buffers in plasma, bicarbonate, per se, would considerably decrease $\mathrm{pHi}$ although $\mathrm{pHa}$ may increase due to the readily entrance of $\mathrm{CO}_{2}$ (but not - $\mathrm{HCO}_{3}$ ) into intracellular spaces [56]. Nevertheless, severe acidosis $(\mathrm{pHa}<7.1)$ must be timely corrected at any clinical circumstances although mild acidosis may be beneficial. Without an ideal base in the clinic setting, it is strongly recommended that the bicarbonate infusion should be cautious in the management of cardiopulmonary arrest in order to maintain $\mathrm{pHa}$ in 7.10-7.20 after the airway is established [57]. Clinical evidence also revealed that bicarbonate therapy provided little improvement of diabetic ketoacidosis and suggested that it should not be used unless pHa is $<7.0[19,58]$. On the other hand, DCA, which stimulates the PDC activity by directly inhibiting PDC kinase as one of Pyr actions and corrects lactic acidosis as a specific agent, also can significantly improve myocardial performance and acidosis in hypoxia [14, 20]. In comparison, Pyr may be superior to bicarbonate or DCA in the treatment of hypoxic acidosis. Its unique metabolic and chemical characteristics may be ideal for the correction of severe acidosis in cardiogenic shock or hypercapnia with cardiac arrest as it consumes protons and benefits in myocardium performance without risking $\mathrm{pHi}$ deterioration in anaerobic conditions. Further, Pyr protects cellular functions of multiple cell lines and vital organs as a strong antioxidant. It also inhibits inflammation mediators (e.g., TNF- $\alpha$, NF$\kappa \mathrm{B}$ and $\mathrm{NO}$ ), improves $\mathrm{Ca}^{2+}$ homeostasis, attenuates apoptosis and preserves carbohydrates metabolic pathways in hyperglycemia and promotes lipid metabolism $[2,3,5,6,52]$. Pyr as an intermediary metabolite of glucose is also an energy-yielding substrate without any known cytotoxicity. An intravenous Pyr loading test demonstrated its safety in human subjects [59].

In addition, Pyr might be also a promising buffer to correct uremic acidosis in patients undergoing peritoneal dialysis (PD), which markedly improves the biocompatibility of PD solutions [5, 36]. It may efficiently correct chronic acidotic status in hemodialysis patients as well, which is not satisfactorily treated at present [60]. Pyr as one of the buffer components may decrease bicarbonate or Lac concentrations in dialysis solutions, reduce oxidative stress induced by high bicarbonate levels [61] and Lac toxicity. It can also improve glucose and lipid metabolism in dialysis patients particularly with cardiovascular complications and diabetes. However, the instability of sodium Pyr in aqueous solutions restricts its clinical applications at the present time. It is well known that Pyr in aqueous solutions rapidly undergoes an aldol-like condensation, forming parapyruvate to inhibit mitochondrial functions [62]. Efforts to overcome Pyr instability in solutions creating Pyr therapeutic preparations suitable for intravenous infusion and dialysis are urgently warranted. Several clues point to a possibility.

In conclusion, Pyr as a novel generation of buffers would be superior to conventional ones both in animal experiments and in clinic settings, particularly in the 
treatment of severe acidemia, which still lacks effective agents to deal with at present. Intravenous administration of Pyr would not only correct both $\mathrm{pHa}$ and $\mathrm{pHi}$ and acidotic symptoms, but also improve underlying cell dysfunctions to a large extent in critically ill patients with heart, liver, kidney or brain disorders. Further evaluation of Pyr effects on acid-base balance disorders and their underlying mechanisms in both animals and clinical trials is required. Attempts to create stable therapeutic preparations of Pyr solutions are warranted.

\section{Acknowledgements}

The author is indebted to Dr. Z.D. Zhou at St. Joseph Medical Center, Syracuse, N.Y. for his help in the improvement of the manuscript. The author also thanks the reviewers for their critical comments.

\section{References}

-1 Hermann H-P, Pieske B, Schwarzmuller E, Keul J, Just H, Hasenfuss G: Haemodynamic effects of intracoronary pyruvate in patients with congestive heart failure: An open study. Lancet 1999;353:1321-1323.

2 Kristo G, Yoshimura Y, Niu J, Keith BJ, Mentzer RM Jr, Bunger R, Lasley RD: The intermediary metabolite pyruvate attenuates stunning and reduces infarct size in in vivo porcine myocardium. Am J Physiol 2004;286:H517H524.

3 Lopaschuk GD, Rebeyka IM, Allard MF: Metabolic modulation: A means to mend a broken heart. Circulation 2002;105:140-142.

-4 Ing TS, Zhou XJ, Yu AW, Zhou FQ, Vaziri ND: Effects of pyruvate-based or lactate-based peritoneal dialysis solutions on neutrophil intracellular pH. Int J Artif Organs 1997;20:255260.

5 Zhou FQ: Advantages of pyruvate over lactate in peritoneal dialysis solutions. Acta Pharmacol Sin 2001;22:385-392.

-6 Mongan PD, Karaian J, Van Der Schuur BM, Via DK, Sharma P: Pyruvate prevents polyADP ribose polymerase (PARP) activation, oxidative damage, and pyruvate dehydrogenase deactivation during hemorrhagic shock in swine. J Surg Res 2003;112:180-188.

$\checkmark 7$ Schwartz WB, Waters WC 3rd: Lactate versus bicarbonate: A reconsideration of the therapy of metabolic acidosis. Am J Med 1962;32:831834.

$\checkmark 8$ McLean AG, Davenport A, Cox D, Sweny P: Effects of lactate-buffered and lactate- free dialysate in CAVHD patients with and without liver dysfunction. Kidney Int 2000;58:17651772.

9 Cohen RD: Roles of the liver and kidney in acid-base regulation and its disorders. Br J Anaesth 1991;67:154-164.

10 Griffin JL, White LT, Lewandowski ED: Substrate-dependent proton load and recovery of stunned hearts during pyruvate dehydrogenase stimulation. Am J Physiol 2000;279:H361H367.
11 Hasenfuss G, Maier LS, Hermann H-P, Luers C, Hunlich M, Zeitz O, Janssen PM, Pieske B: Influence of pyruvate on contractile performance and $\mathrm{Ca}(2+)$ cycling in isolated failing human myocardium. Circulation 2002;105: 194-199.

12 Samaja M, Allibardi S, Milano G, Neri G, Grassi B, Gladden LB, Hogan MC: Differential depression of myocardial function and metabolism by lactate and $\mathrm{H}^{+}$. Am J Physiol 1999; 276:H3-H8.

13 Zubay GL, Parson WW, Vance DE: The tricarboxylic acid cycle; in Zubay GL, Parson WW, Vance DE (eds): Principles of Biochemistry, Chapt 13. Dubuque, Brown Communications Inc, 1995, pp 282-304.

14 Dodgson SJ, Forster RE 2nd: Inhibition of CA $\mathrm{V}$ decreases glucose synthesis from pyruvate Arch Biochem Biophys 1986;251:198-204.

15 Filippi L, Bagnoli F, Margollicci M, Zammarchi E, Tronchin M, Rubaltelli FF: Pathogenic mechanism, prophylaxis, and therapy of symptomatic acidosis induced by acetazolamide. $\mathrm{J}$ Investig Med 2002;50:125-132.

16 Stacpoole PW, Nagaraja NV, Hutson AD: Efficacy of dichloroacetate as a lactate- lowering drug. J Clin Pharmacol 2003;43:683-691.

17 Healey MA, Davis RE, Liu FC, Loomis WH, Hoyt DB: Lactated Ringer's is superior to normal saline in a model of massive hemorrhage and resuscitation. J Trauma 1998;45:894899.

18 Yu AW, Zhou XJ, Zhou FQ, Nawab ZM, Gandhi VC, Ing TS, Vaziri ND: Neutrophilic intracellular acidosis induced by conventional, lactate-containing peritoneal dialysis solutions. Int J Artif Organs 1992; 15:661-665.

19 Kraut JA, Kurtz I: Use of base in the treatment of severe acidemic states. Am J Kidney Dis 2001;38:703-727.

20 Stacpoole PW, Wright EC, Baumgartner TG, et al: A controlled clinical trial of dichloroacetate for treatment of lactic acidosis in adults. N Engl J Med 1992;327:1564-1569.

21 Kline JA, Thornton LR, Lopaschuk GD, Barbee RW, Watts JA: Lactate improves cardiac efficiency after hemorrhagic shock. Shock 2000;14:215-221.
-22 Kline JA, Maiorano PC, Schroeder JD, Grat$\tan$ RM, Vary TC, Watts JA: Activation of pyruvate dehydrogenase improves heart function and metabolism after hemorrhagic shock. J Mol Cell Cardiol 1997;29:2465-2474.

$\checkmark 23$ Swafford AN Jr, Bidros D, Truxillo TM, Giaimo ME, Miller HI, McDonough KH: Ethanol intoxication and lactated Ringer's resuscitation prolong hemorrhage-induced lactic acidosis. Shock 2003;20:237-244.

24 Lloyd SG, Wang P, Zeng H, Chatham JC: Impact of low-flow ischemia on substrate oxidation and glycolysis in the isolated perfused rat heart. Am J Physiol 2004;287:H351-H362.

25 Bailey SM, Reinke LA: Effect of low flow ischemia-reperfusion injury on liver function. Life Sci 2000;66:1033-1044.

-26 Bellomo R: Bench-to-bedside review: Lactate and the kidney. Crit Care 2002;6:322-326.

27 Cano N: Bench-to-bedside review: Glucose production from the kidney. Crit Care 2002;6: 317-321.

28 Kondou I, Nakada J, Hishinuma H, Masuda F, Machida T, Endou H: Alterations of gluconeogenesis by ischemic renal injury in rats. Ren Fail 1992; 14:479-483.

29 Hwang JJ, Curthoys NP: Effect of acute alternations in acid-base balance on rat renal glutaminase and phosphoenolpyruvate carboxykinase gene expression. J Biol Chem 1991;266: 9392-9396.

30 Chiolero RL, Revelly JP, Leverve X, Gersbach P, Cayeux MC, Berger MM, Tappy L: Effects of cardiogenic shock on lactate and glucose metabolism after heart surgery. Crit Care Med 2000;28:3784-3791.

-31 Koustova E, Rhee P, Hancock T, Chen H, Inocencio R, Jaskille A, Hanes W, Valeri CR, Alam HB: Ketone and pyruvate Ringer's solutions decrease pulmonary apoptosis in a rat model of severe hemorrhagic shock and resuscitation. Surgery 2003;134:267-274.

32 Liedtke AJ, Nellis SH, Neely JR, Hughes HC: Effects of treatment with pyruvate and tromethamine in experimental myocardial ischemia. Circ Res 1976;39:378-387. 
-33 Yanos J, Patti MJ, Stanko RT: Hemodynamic effects of intravenous pyruvate in the intact, anesthetized dog. Crit Care Med 1994;22:844850 .

34 Mongan PD, Fontana JL, Chen R, Bunger R: Intravenous pyruvate prolongs survival during hemorrhagic shock in swine. Am J Physiol 1999;277:H2253-H2263.

35 Zhou FQ: Pyruvate improvement of cellular energetics, not simply generating ATP during anoxia. Transplant 2002;73:1851.

>36 Wu YT, Wu ZL, Jiang XF, Li S, Zhou FQ: Pyruvate preserves neutrophilic superoxide production in acidic, high glucose-enriched peritoneal dialysis solutions. Artif Organs 2003;27: 276-281.

-37 Regitz V, Azumi T, Stephan H, Naujocks S, Schaper W: Biochemical mechanism of infarct size reduction by pyruvate. Cardiovasc Res 1981;15:652-658.

- 38 Satoh H, Sugiyama S, Nomura N, Terada H, Hayashi $\mathrm{H}$ : Importance of glycolytically derived ATP for $\mathrm{Na}^{+}$loading via $\mathrm{Na}^{+} / \mathrm{H}^{+}$exchange during metabolic inhibition in guinea pig ventricular myocytes. Clin Sci 2001;101: 243-251.

-39 Karwatowska-Prokopczuk E, Nordberg JA, Li HL, Engler RL, Gottlieb RA: Effect of vacuolar proton ATPase on $\mathrm{pH}_{\mathrm{i}}, \mathrm{Ca}^{2+}$, and apoptosis in neonatal cardiomyocytes during metabolic inhibition/recovery. Circ Res 1998;82:11391144.

-40 Sileri P, Schena S, Morini S, Rastellini C, Pham S, Benedetti E, Cicalese L: Pyruvate inhibits hepatic ischemia-reperfusion injury in rats. Transplantation 2001;72:27-30.

41 Mongan PD, Capacchione J, Fontana JL, West S, Bunger R: Pyruvate improves cerebral metabolism during hemorrhagic shock. Am J Physiol 2001;281:H854-H864.

42 Mongan PD, Capacchione J, West S, Karaian J, Dubois D, Keneally R, Sharma P: Pyruvate improves redox status and decreases indicators of hepatic apoptosis during hemorrhagic shock in swine. Am J Physiol 2002;283:H1634H1644.
43 Saiki Y, Lopaschuk GD, Dodge K, Yamaya K, Morgan C, Rebeyka IM: Pyruvate augments mechanical function via activation of the pyruvate dehydrogenase complex in reperfused ischemic immature rabbit hearts. J Surg Res 1998;79:164-169.

44 Panchal AR, Comte B, Huang H, Kerwin T, Darvish A, des Rosiers C, Brunengraber $\mathrm{H}$, Stanley WC: Partitioning of pyruvate between oxidation and anaplerosis in swine hearts. Am J Physiol 2000;279:H2390-H2398.

45 Oliver FJ, Salto R, Sola MM, Vargas AM: Mitochondrial pyruvate metabolism in liver and kidney during acidosis. Cell Biochem Funct 1994;12:229-235.

46 Burns SP, Murphy HC, Iles RA, Cohen RD: Lactate supply as a determinant of the distribution of intracellular $\mathrm{pH}$ within the hepatic lobule. Biochem J 2001;358:569-571.

47 Jackson VN, Halestrap AP: The kinetics, substrate, and inhibitor specificity of the monocarboxylate (lactate) transporter of rat liver cells determined using the fluorescent intracellular $\mathrm{pH}$ indicator, $2^{\prime}, 7^{\prime}$-bis(carboxyethyl)5(6)- carboxyfluorescein. J Biol Chem 1996; 271:861-868.

48 Wang X, Levi AJ, Halestrap AP: Kinetics of the sarcolemmal lactate carrier in single heart cells using BCECF to measure pHi. Am J Physiol 1994;267:H1759-H1769.

49 Martin BJ, Valdivia HH, Bunger R, Lasley RD, Mentzer RM Jr: Pyruvate augments calcium transients and cell shortening in rat ventricular myocytes. Am J Physiol 1998;274:H8H17.

50 Wathen RL, Ward RA, Harding GB, Meyer LC: Acid-base and metabolic responses to anion infusion in the anesthetized dog. Kidney Int 1982;21:592-599.

$>51$ Berry MN, Phillips JW, Gregory RB, Grivell AR, Wallace PG: Operation and energy dependence of the reducing-equivalent shuttles during lactate metabolism by isolated hepatocytes. Biochim Biophys Acta 1992;1136:223-230.

52 Lee YJ, Kang IJ, Bunger R, Kang YH: Enhanced survival effect of pyruvate correlates MAPK and NF- $\mathrm{B}$ activation in hydrogen peroxide-treated human endothelial cells. J Appl Physiol 2004;96:793-801.
53 Kubasiak LA, Hernandez OM, Bishopric NH, Webster KA: Hypoxia and acidosis activate cardiac myocyte death through the Bcl-2 family protein BNIP3. Proc Natl Acad Sci USA 2002;99:12825-12830.

54 Olivencia-Yurvati AH, Blair JL, Baig M, Mallet RT: Pyruvate-enhanced cardioprotection during surgery with cardiopulmonary bypass. J Cardiothorac Vasc Anesth 2003; 17:715720 .

55 Mizock BA, Falk JL: Lactic acidosis in critical illness. Crit Care Med 1992;20:80-93.

56 Levraut J, Giunti C, Ciebiera J-P, de Sousa G, Ramhani R, Payan P, Grimaud D: Initial effect of sodium bicarbonate on intracellular $\mathrm{pH}$ depends on the extracellular non-bicarbonate buffering capacity. Crit Care Med 2001;29: 1033-1039.

57 National Conference on Cardiopulmonary Resuscitation: Standards and guidelines for cardiopulmonary resuscitation and emergency cardiac care. Part III: Adult advanced cardiac life support. JAMA 1986;255:2933-2954.

58 Chiasson J-L, Aris-Jilwan N, Belanger R, Bertrand $\mathrm{S}$, Beauregard $\mathrm{H}$, Ekoe J-M, Fournier $\mathrm{H}$, Havrankova, J: Diagnosis and treatment of diabetic ketoacidosis and the hyperglycemic hyperosmolar state. CMAJ 2003;168:859866.

-59 Van Erven PMM, Gabreels FJM, Wevers RA, Doesburg WH, Ruitenbeek W, Renier WO, Lamers KJB: Intravenous pyruvate loading test in Leigh syndrome. J Neurol Sci 1987;77: 217-227.

60 Kovacic V, Roguljic L, Kovacic V: Metabolic acidosis of chronically hemodialyzed patients. Am J Nephrol 2003;23:158-164.

61 Epperlein MM, Nourooz-Zadeh J, Jayasena SD, Hothersall JS, Noronha-Dutra A, Neild $\mathrm{GH}$ : Nature and biological significance of free radicals generated during bicarbonate hemodialysis. J Am Soc Nephrol 1998;9:457-463.

62 Fink MP: Ringer's ethyl pyruvate solution: A novel resuscitation fluid. Minerva Anestesiol 2001;67:190-192. 